\title{
Research on the Factors Affecting the Communication Effect of Scene Music Song Lists
}

\section{${ }^{1}$ Lu Jiang, ${ }^{2}$ Wenying Wu}

${ }^{1}$ Division of communication, Faculty of Humanities and Social Sciences, Dalian University of Technology, Dalian, Liaoning, China. 2Division of communication, Faculty of Humanities and Social Sciences, Dalian University of Technology. Dalian, Liaoning, China.

Correspondence Author: Lu Jiang, Division of communication, Faculty of Humanities and Social Sciences, Dalian University of Technology, Dalian, Liaoning, China

Received date: 12 May 2019, Accepted date: 24 September 2019, Online date: 25 October 2019

Copyright: (c) 2019 Lu Jiang and Wenying Wu. This is an open-access article distributed under the terms of the Creative Commons Attribution License, which permits unrestricted use, distribution, and reproduction in any medium, provided the original author and source are cre dited.

\begin{abstract}
In the era of mobile communication, the scene has become one of the core elements of the media. The combination of music and life scenes has also become the development trend of online music apps. The scene mainly refers to the virtual scene constructed by mobile technology, and its extension, interaction and behavior in the real space, and the psychological environment. This paper uses quantitative research methods such as Multi-layer regression analysis, to analyze the relationship between scenes and song lists. In this study, the relationship between the communication bias of the title, picture type, the number of songs, release time of the song lists, the scene of the song lists, the experience of the song maker, and the relationship between the effects of the songs and the songs of the scene are analyzed. The results show that: 1 . The song lists type is positively correlated with the listening effect and interaction effect, but there is no significant predictive power. 2 . Title propagation bias, picture type, and release time have significant predictive power for interactive effects. 3 , the picture is related to the title, and the release of the song lists has a significant predictive power for the listening effect. 4. There is a significant positive correlation between the song lists type and the title propagation bias. 5 , song lists scene type has a certain impact on the number of songs, song language.
\end{abstract}

Keywords: scene, song lists, communication effect

\section{INTRODUCTION}

1.1 The research background

According to the "41st Statistical Report on Internet Development in China", as of December 2017, the number of users of online music in China reached 548.09 million, and the usage rate of Internet users was 71\%. (China Internet Information Center, 2018)

Online music app, the scene song list is now widely available. QQ Music, Net Ease Cloud music, etc. combine music and life scenes, and launched the "running FM" function. The online music app pushes song lists and songs based on listening to music scenes such as rest and work.

Professor Lan Peng [1] said that in the era of mobile communication, the scene became one of the core elements of the media. It is important to study the communication of scene music.

\subsection{Literature review}

\subsubsection{Related research on scene connotation}

The concept of the scene is presented in Merovitz's The Disappearing Region: The Impact of Electronic Media on Social Behavior (2002). The word situation, the introduction of the electronic media and the "information scene" created by it, transcends the boundaries of the material field, is a mode of information flow. This concept is based on the era of electronic media, pointing out the information composition and flow of the scene, the virtuality of the scene and the characteristics of transcending boundaries.

The context of the upcoming scene era (2014) by [2] uses context to describe the scene, which refers to the scene mainly represented by the virtual scene constructed by mobile technology, and it is in real space: extension, interaction. At the same time, they proposed five elements related to the scene era: big data, mobile devices, social media, sensors, positioning systems, and called it "scenes five forces".

Professor Lan Peng [1] of Tsinghua University said that the scene can cover space-based and behavior-based, psychological environment. The basic elements of the scene should be space and environment, user real-time status, user life inertia, social atmosphere. The essence of mobile communication is the scene-based service, that is, the perception of the scene and the adaptation of information.

Scene music originally referred to as music used in a single scene in a movie or television, which only works for a specific scene. In the era of scene communication, scene music refers to a type of music that occurs in people's living space and can satisfy the music needs of users in real or virtual scenes [3] 
Citation: Lu Jiang and Wenying Wu, 2019. Research on the Factors Affecting the Communication Effect of Scene Music Song Lists. Journal of Applied Sciences Research., 15(5): 1-6. DOI: 10.22587/jasr.2019.15.5.1

\subsubsection{Study on music transmission of scenario \\ -theoretical research on music transmission in scenario}

Ethnomusicologist John blacking [4] says that music brings people into a powerful shared experience. The sharing of musical experience is often combined with our life scenes and is connected with our daily life. Under the mobile technology, the communication and interaction between virtual scene and reality scene brought by music APP.

Qing Peng [5] music fans are both consumers of scenes and producers of scenes. Fans deconstruct traditional scenes and reconstruct new scenes. When music relies on scenes to move towards real space, the relationship between music and human beings is reconstructed by the scene.

Users' real-time status is not only reflected in their own data, but also in the environmental information they are interested in [1]. Therefore, the music user's scene state actually contains two levels: psychological emotion and information. The dissemination of music is also divided into the transmission of emotional flow and the dissemination of information flow.

Therefore, the relationship between music message demand expectations, search habits, and music preferences, etc., caused by the title of the UGC scene song list, and the scene variables are worthy of investigation.

Therefore, the hypothesis is put forward:

H1, the communication bias of the title is related to the scene type

H2, the communication bias of title is related to the communication effect

\section{-exploration of the research method of scenario music communication}

Scholar Mill ward, P [6] argue that social network analysis is a useful way to explore music scenes. Through the discovery of Britpop, it can be summarized as the accumulation of other music scenes, cultural labor and youth, and study the relationship between them.

The scholar Driver, Christopher [7] believes that the tangible elements of the scene revealed by the embodiment of social actors are the key to understanding of music scenes.

Scholars Ironi, Manuel [8] provided a mobile, short-lived and fluid approach to the localized (cultural) economy in Chile based on experimental music scenes in Santiago. It also provides a new perspective on cultural clusters under the study of music scenes.

Lan Peng [1] pointed out that the mobile scene refers to the environment that people are constantly encountering in their activities and is a "variable". For each specific user, moving the scene means the moment of fast switching, and each scene brings different needs.

It can be seen that the study of the propagation effect of the scene as a variable is of great significance.

\section{Therefore:}

Research question 1: the relationship between scene type and picture type, picture and title related, number of songs, song language, song lists release time from now, song lists description and other variables

Research question 2: the relationship between communication effect and picture type, picture and title related, number of songs, song language, song lists release time from now, and description of song lists

H3, the type of scene is related to the communication effect

\section{-scenario music communication process and social impact analysis}

Scholar Spring, Kenneth [9] analyzes risk seeking and risk negotiation in music carnival scenarios.

Scholar Taylor, Jodie [10] describes the integration of queer theory in music scenes and demonstrates the need for "scene" to understand the queer group in everyday music.

While mobile terminals change the media usage scenarios in the home, they also influence the relationship of family members [1]. It can be seen that the scene music distribution not only reconstructs the scene where the user is located, but also affects the social relationship of the user.

The role of user factors in the spread of scene music has not yet been explored. This study intends to explore this.

H4, the experience of song lists producer is related to the communication effect

\subsection{Research design}

\subsubsection{The Sampling}

Net Ease Cloud music user activity and market share are the fourth in the Chinese market, and the elements of the song list are complete. Therefore, the song list of Net Ease Cloud Music was selected as the research object.

The analysis unit of this study is a single scene music song list. The research object is the scene music song list. The current scenes commonly used in the industry are classified into five categories: night (sleeping/night), learning, work, travel, and sports.

Therefore, the sample of this study is a total of 6862 popular scene music songs in Net Ease Cloud Music. According to the calculation, when the confidence is $95 \%$ and the confidence interval is 5.5 , the sample size is 303 . To facilitate the collection, set the sample size to 300 . Take stratified sampling, equidistant sampling, and take samples.

\subsubsection{Category construction}

The first-level indicators of this study are song-single communication effect, song list content, song list maker.

The secondary indicators are number of plays, times of forwarding, number of favourites, number of comments, basic information of song list, song form, song content, user level, cumulative number of listening songs, number of song lists produced, number of song list to be collected.

Three-level indicator:

Song lists release time: (1) within 3 months, (2) within half a year, (3) within one year, (4) within two years, (5) more than two years.

Scene types :(1) work, (2) study, (3) travel, (4) exercise, (5) night.

Description :(1) no, (2) yes

Title communication bias :(1) no emotion or information; (2) information bias; (3) emotion bias; (4) both emotion and information

Picture type :(1) other, (2) emojis, (3) animals, (4) scenery, (5) anime, (6)human.

Pictures and title related: (1) no, (2) yes.

Song language: (1) pure music, (2) other, (3) Korean, (4) Japanese, (5) English, (6) Chinese.

\subsubsection{Pretest}

The research content analysis was carried out by two coders. In terms of reliability calculation, Hosti [11] scorer reliability method was used, and the reliability coefficient was 0.944 , which accorded with the level of reliability.

\section{Data Analysis}

\subsection{Sample structure description}

This study analyzed 300 music song lists. There are $30 \%(\mathrm{n}=90)$ sing lists issued within three months, 16.7\% $(\mathrm{n}=50)$ in six months, 15\% $(\mathrm{n}=45)$ in one year, $19 \%(\mathrm{n}=57)$ in two years and $19.3 \%(\mathrm{n}=58)$ in more than two years.

Song lists of five scene types, released within 3 months, accounted for a large proportion.

The number of song lists with description was 248 , accounting for 82.7 percent, while the number of songs without description was 52 , accounting for 17 percent. In the five types of scenes, the playlists with descriptions were all higher than the playlists without descriptions. 
The title of the song lists mainly conveys information $(\mathrm{n}=182,60.7 \%)$, and $20.3 \%(\mathrm{n}=61)$ convey emotion, and $17.7 \%$ ( $\mathrm{n}=53)$ contain both emotion and information. Rarely, the title of the song lists is neither emotion nor information $(n=4,1.3 \%)$.

In the four scenes of work, study, travel and sports, the information dissemination of the song title is more prominent. The night scene, for the moment of relaxation and relaxation, the title of the song lists has the tendency of spreading emotion, as well as both passion and information. (Figure 1)

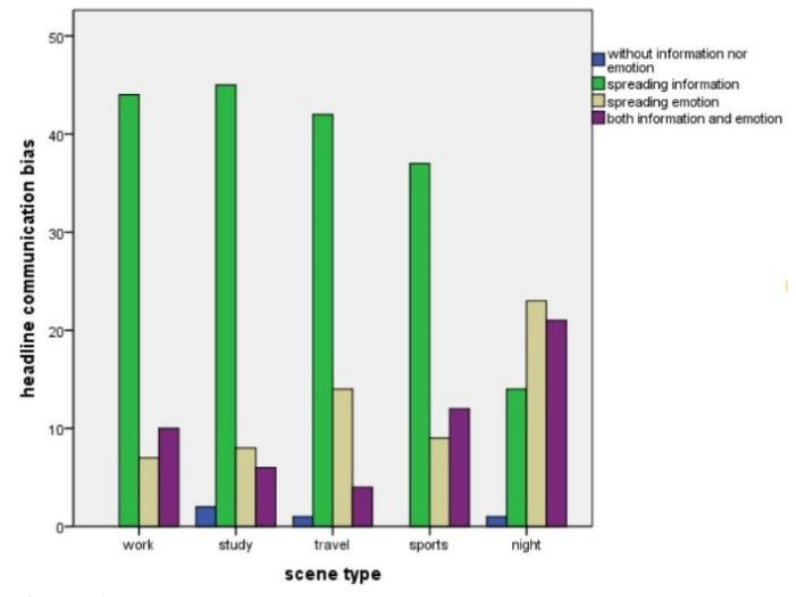

Figure 1: scene type \& title communication bias

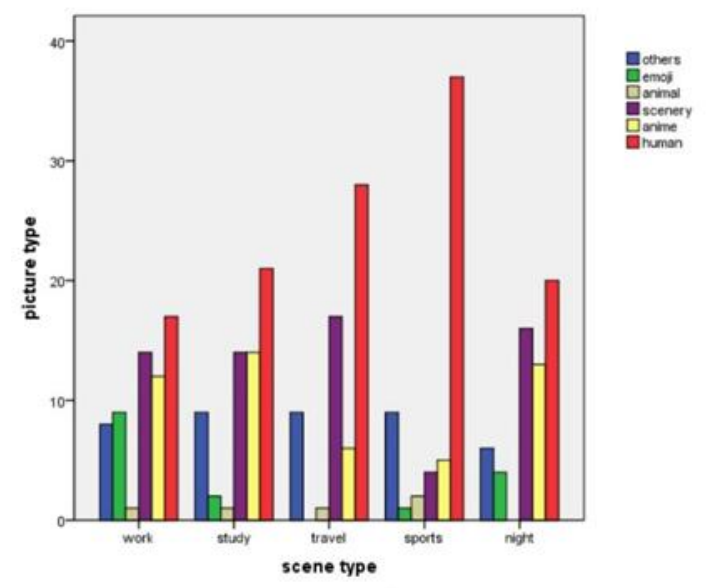

Figure 2: scene type \& picture type

Song lists cover image is given priority to with human $(n=1239 \%)$, followed by the cover of the scenery picture $(n=65,21.6 \%)$, cartoon images accounted for $16.7 \%(\mathrm{n}=50)$, other types of images including posters, LOGO, design class vector diagram accounted for $13.7 \%(\mathrm{n}=41)$, such as song single package cover photo for expression of $5.3 \%(\mathrm{n}=16)$, is the least amount of animal images accounted for $1.7 \%(\mathrm{n}=5)$.

Figure pictures account for the largest number of the five scenes, especially sports. Exercise and fitness are all related to the human body, and the image of the human body shows the beauty of health. (figure 2)

The image content of song lists is related to the title, accounting for $59.7 \%(n=179)$, while the image content is unrelated to the title, accounting for $40.3 \%$ $(\mathrm{n}=121)$.

The median number of songs in each song lists is 61 , the average is 158.97 , the maximum is 8454 , the minimum is 9 , and the standard deviation is 536.1 . English songs are also included in 143 song lists, 116 song lists contain pure music and 111 song lists contain Chinese songs. There are 38 songs in Japanese, 10 in Korean and 7 in other minor languages.

Most song lists contain songs in one language $(\mathrm{n}=218)$ and 59 song lists contain music in two languages. There are 13 song lists for 3 languages and 10 song lists for 4 languages.

The average user level of song lists producers is 7.68 , and the average cumulative number of songs listening recorded by a song lists producer is 4,815 , the average number of songs produced is 35.8 pieces per person, and the average number of song lists collection is 76.1 pieces.

\subsection{Research and analysis}

In this study, factor analysis was performed on the effect variables of song lists communication (playing times, forwarding times, collection Numbers and comments). The KMO value was 0.513 , the approximate chi-square of Bartlett test was 1429.97 , and $\mathrm{P}<0.001$ met the condition of factor analysis. Two factors were extracted and named as listening effect and interaction effect.

Factor analysis was performed on the variables (user level, the cumulative number of songs heard, number of song lists production, and collection number) of the producer of song lists. KMO was 0.695, and the approximate chi-square of Bartlett test was 147.17. P<0.001 met the condition of factor analysis. A factor is extracted and named as the playlist maker's experience.

There was a positive correlation between the title communication bias and the type of night scene $\left(\chi^{2}=0.338, \mathrm{P}<0.01 ;\right.$ Spearman $\left.=0.355, \mathrm{P}<0.01\right)$.

There was a negative correlation between the title communication bias and the type of learning scene $\left(\chi^{2}=-0.158, \mathrm{P}<0.01 ;\right.$ Spearman $\left.=-0.164, \mathrm{P}<0.01\right)$.

There is a negative correlation between the communication bias of the song lists title and the type of the travel scene $\left(\chi^{2}=-0.127, \mathrm{P}<0.05\right)$.

There was a positive correlation between title communication bias and scene type $\left(\chi^{2}=0.276, \mathrm{P}<0.01 ;\right.$ Spearman $\left.=0.291, \mathrm{P}<0.01\right)$.

Therefore, research hypothesis 1 is supported.

There was a positive correlation between title bias and listening effect $\left(\chi^{2}=0.121 *, \mathrm{P}<0.05\right.$; Spearman $\left.=0.149 * *, \mathrm{P}<0.01\right)$, and interaction effect $\left(\chi^{2}=\right.$ $0.137 *$, Spearman $=0.181 * *, \mathrm{P}<0.01)$. Hypothesis 2 is supported. 
Citation: Lu Jiang and Wenying Wu, 2019. Research on the Factors Affecting the Communication Effect of Scene Music Song Lists. Journal of Applied Sciences Research., 15(5): 1-6. DOI: 10.22587/jasr.2019.15.5.1

Figure 3 scene type $\&$ variables

\begin{tabular}{|l|l|l|l|l|l|l|}
\hline & work & study & travel & sports & night & Scene type \\
\hline & $\rho$ & $\rho$ & $\rho$ & $\rho$ & $\rho$ & $\chi^{2}$ \\
\hline Title bias & -0.079 & $-0.164^{* *}$ & -0.113 & 0.005 & $0.355^{* *}$ & $0.276^{* * 1}$ \\
\hline Picture type & $-0.128^{*}$ & -0.035 & 0.029 & $0.165^{* *}$ & -0.028 & 0.101 \\
\hline Picture\& title related & -0.091 & 0.027 & $-0.176^{* *}$ & 0.058 & $0.185^{* *}$ & $0.164^{* *}$ \\
\hline song number & 0.025 & 0.056 & 0.040 & -0.005 & $-0.118^{*}$ & $-0.138^{*}$ \\
\hline language & -0.097 & -0.067 & 0.089 & 0.063 & 0.014 & 0.104 \\
\hline Release time & $0.122^{*}$ & -0.059 & 0.005 & 0.065 & $-0.134^{*}$ & $-0.114^{*}$ \\
\hline description & -0.053 & $0.122^{*}$ & -0.097 & -0.088 & $0.116^{*}$ & 0.036 \\
\hline Statistically significant value & & $* \mathrm{P}<0.05$ & $* * \mathrm{P}<0.01$ & $* * * \mathrm{P}<0.001$ \\
\hline
\end{tabular}

Image type and work scene have a negative correlation (Spearman $=-0.128^{*}$ ).

There is a significant positive correlation between picture type and sports scene $\quad\left(\right.$ Spearman $\left.=0.165^{* *}\right)$

There is a significant positive correlation between Picture \& Title related and night scene $($ Spearman $=0.185, \mathrm{P}<0.01)$.

Picture \& Title Related associated with travel scene, has significant negative correlation $($ Spearman $=0.176, \mathrm{P}<0.01$ )

Picture \& Title Related is relate with the scene, has significant positive correlation $\left(\chi^{2}=0.164, \mathrm{P}<0.164\right)$

Number of song lists and the night scene has a negative correlation (Spearman $=0.118, \mathrm{P}<0.05)$

Number of song lists and the scene type has a negative correlation $\left(\chi^{2}=0.138, P<0.05\right)$

English and night scene have significant negative correlation $($ Spearman $=0.187, \mathrm{P}<0.01$ )

English and sports scene have significant positive correlation Spearman $=0.293, \mathrm{P}<0.293$ )

Pure music and sports scene have a negative correlation (Spearman $=0.146, \mathrm{P}<0.05$ )

Pure music and travel scene have a negative correlation (Spearman $=0.129, \mathrm{P}<0.05$ )

Song lists release time from now and night scene have a negative correlation $($ Spearman $=0.134, \mathrm{P}<0.05)$

Song lists release time from now and work scene have the positive correlation between $($ Spearman $=0.122, \mathrm{P}<0.05)$

Song lists release time from now with the scene type have a negative correlation between $\left(\chi^{2}=0.114, \mathrm{P}<0.05\right)$

There is a positive correlation between the description and the type of night scene (Spearman $=0.116, \mathrm{P}<0.05)$.

There is a positive correlation between description and learning scene type (Spearman $=0.122, \mathrm{P}<0.05$ ).

Research question 1 is verified. (Figure 3)

Research question 2: the relationship between communication effect and picture type, Picture \& Title Related, number of songs, song language, the release time of song lists, and description of song lists. (Figure 4, 5)

Figure 4 Listening effect

\begin{tabular}{|c|c|c|c|c|c|c|}
\hline & \multicolumn{2}{|c|}{ Model 1 } & \multicolumn{2}{c|}{ Model 2 } & \multicolumn{2}{c|}{ Model 3 } \\
\hline & $\beta$ & SE & $\beta$ & SE & $\beta$ & SE \\
\hline release time & $0.193 * * *$ & 0.037 & $0.172 * * *$ & 0.038 & $0.150 * * *$ & 0.039 \\
\hline Scene type & 0.061 & 0.039 & 0.023 & 0.042 & 0.019 & 0.042 \\
\hline Title bias & & & 0.113 & 0.073 & 0.109 & 0.073 \\
\hline Picture type & & & 0.030 & 0.032 & 0.029 & 0.032 \\
\hline Picture \& Title Related & & & $0.241 *$ & 0.117 & $0.240 *$ & 0.116 \\
\hline Song number & & & $-8.920 \mathrm{E}-005$ & 0.000 & 0.000 & 0.000 \\
\hline language & & & -0.026 & 0.028 & -0.024 & 0.028 \\
\hline Description & & & 0.213 & 0.149 & 0.205 & 0.148 \\
\hline Producers' experience & & & & & 0.112 & 0.060 \\
\hline AdjustedR & & & 0.104 & & 0.111 & \\
\hline F & 0.081 & & 5.29 & & 5.14 & \\
\hline Statistically significant value & & $* \mathrm{P}<0.05$ & $* * \mathrm{P}<0.01$ & $* * * \mathrm{P}<0.001$ & & \\
\hline
\end{tabular}

Model 1 studies the predictive power between the basic information (release duration, scene type) and listening effect. Among them, there is a significant predictive force between release duration and listening effect $(\beta=0.193 * *, S E=0.037, P<0.001)$, and there is no vital predictive force for scene type $(\beta=0.061$, $\mathrm{SE}=0.039, \mathrm{P}>0.05)$

Model 2 studies the predictive power between the basic information, content and listening effect of the song lists. Among them, the picture content is related to the title and has a significant predictive power between the listening effect and the listening effect $\left(\beta=0.241^{*}, \mathrm{SE}=0.073\right.$, $\left.\mathrm{P}<0.05\right)$, and there is no significant predictive power for the title communication bias, picture type, playlist description, number of songs, and language of songs.

Model 3 studies the predictive power between the basic information, the content, the producer's experience and the listening effect. Among them, the timing of song lists release and listening effect still have significant predictive power $(\beta=0.150 * * *, \mathrm{SE}=0.039, \mathrm{P}<0.001)$.

The image content which is related to the title is still significantly predictive of listening performance $(\beta=0.240 *, \mathrm{SE}=0.116$, $\mathrm{P}<0.05)$. There was no significant predictive power of title communication bias, picture type, album description, number of songs, the language of songs, and producer experience. 
Citation: Lu Jiang and Wenying Wu, 2019. Research on the Factors Affecting the Communication Effect of Scene Music Song Lists. Journal of Applied Sciences Research., 15(5): 1-6. DOI: $10.22587 /$ jasr.2019.15.5.1

\section{Figure5 The interactive effect}

\begin{tabular}{|c|c|c|c|c|c|c|}
\hline & \multicolumn{2}{|c|}{ Model 1} & \multicolumn{2}{|c|}{ Model 2} & \multicolumn{2}{|c|}{ Model 3} \\
\hline & $\beta$ & SE & $\beta$ & SE & $\beta$ & SE \\
\hline release time & 0.062 & 0.038 & $0.084 *$ & 0.039 & $0.089^{*}$ & 0.041 \\
\hline Scene type & 0.022 & 0.041 & 0.010 & 0.043 & 0.011 & 0.044 \\
\hline Title bias & & & $0.192 *$ & 0.076 & $0.193 *$ & 0.076 \\
\hline Picture type & & & $-0.075^{*}$ & 0.033 & $-0.075^{*}$ & 0.033 \\
\hline Picture \& Title Related & & & -0.194 & 0.121 & -0.193 & 0.122 \\
\hline Song number & & & $-2.108 \mathrm{E}-005$ & 0.000 & $-9.475 \mathrm{E}-006$ & 0.000 \\
\hline language & & & 0.043 & 0.029 & 0.042 & 0.029 \\
\hline Description & & & 0.035 & 0.155 & 0.037 & 0.155 \\
\hline Producers' experience & & & & & -0.028 & 0.062 \\
\hline AdjustedR $^{2}$ & 0.003 & & 0.031 & & 0.028 & \\
\hline $\mathrm{F}$ & 1.391 & & 2.189 & & 1.963 & \\
\hline \multicolumn{2}{|c|}{ Statistically significant value } & $* \mathrm{P}<0.05$ & $* * \mathrm{P}<0.01$ & $* * * \mathrm{P}<0.001$ & & \\
\hline
\end{tabular}

Model 1 studies the predictive power between basic information (release duration, scene type) and interaction effect. Among them, there was no significant predictive power for the release duration, scene type and interaction effect.

Model 2 studies the predictive power of basic information, song lists content and interaction effect. Among them, there was significant predictive power between picture type and interaction effect $\left(\beta=-0.075^{*}, \mathrm{SE}=0.033, \mathrm{P}<0.05\right)$. The communication bias and interaction effect of the title have significant predictive power, that is, the more the titlebias and dissemination of information and emotion, the better the interaction effect $(\beta=0.192 *, \mathrm{SE}=0.076, \mathrm{P}<0.05)$. The duration and interaction effect of the song lists showed significant predictive power $\left(\beta=0.084^{*}, \mathrm{SE}=0.039\right.$, $\left.\mathrm{P}<0.05\right)$. Pictures correlated with the title, instructions, number of songs and language of songs were not significantly predictive.

Model 3 studies the predictive power between the basic information of the song lists, the content of the song lists, the experience of the song lists maker and the interaction effect.

Among them, the song lists duration, and there is still a significant predictive power between picture type, Title communication bias and interaction effect.

The scene type of song lists, which adapts to the needs of users in different scenes and different environments, has a significant positive correlation with the listening effect $(\beta=0.181 * *, \mathrm{P}<0.01)$. There was a negative correlation between work scene and listening effect $(\beta=-0.167 * *$, $\mathrm{P}<0.01)$, and a positive correlation between sport scene and listening effect $(\beta=0.122 * *, P<0.05)$.

There is a significant positive correlation between the scene type and interaction effect $\left(\beta=0.185^{* *}\right.$, $\left.\mathrm{P}<0.01\right)$. There was a negative correlation between learning scene and listening effect $(\beta=-0.21 * *, P<0.01)$, and a positive correlation between sports scene and listening effect $(\beta=0.16 * *, P<0.05)$. Hypothesis 3 is supported.

There was a significant predictive power between singer experience and listening effect $(\beta=0.186 * * *, \mathrm{SE}=0.057, \mathrm{P}>0.001)$. That is, the richer the experience of song producers, the better the listening effect of songs. But there is no significant predictive power for the interaction effect. However, songlist makers' experience variables did not show significant predictive power in listening effect model and interactive effect model. Hypothesis 4 was partially supported.

\section{Results and Discussion}

Based on the scene theory, this study studies the influencing factors of the communication effects of the scene music song lists, and draws the following conclusions:

\subsection{The scene type of song lists positively related to listening effect and interaction effect, but it has no significant predictive power.}

The song list scene type is significantly positively correlated with the listening effect and interaction effect. In practice, users in different scenes have different needs for listening to music. Users in similar situations and states have some similarities in physical and psychological states, which can enhance their desire for communication and the space for dialogue.

From the regression model, the scene type variable does not show significant predictive power for the propagation effect. Under the influence of various variables, the influence of scene factors is not direct and significant. This may be due to the choice of the scene, which is a supplementary factor in the user's use of the song list, which has no decisive effect and has no solid influence.

\subsection{Title communication bias, picture type and release duration have significant predictive power for interactive effects.}

The title communication bias always has significant predictive power under the interactive effect multi-layer regression model. It can be seen that the title communication bias of the song list is an important variable that affects the interaction effect of the song list. Increasing the amount of information and emotional components enriched in the title of the song lists can promote user interaction and communication.

The picture type is assigned to the dummy variable, according to the degree of personification of the picture. The image type has a significant predictive effect on the interaction effect. It can be seen that the user's interaction on the song list will be affected by the image anthropomorphic effect. Thus, the visual communication of picture information and emotions has a positive predictive power for the music distribution of the song list and the user interaction effect.

The release of the song list has a significant predictive effect on the interaction effect. In practice, due to the increase in the time of song list release, user usage is also increasing, and UGC content generated by user interaction, such as comments, also accumulates over time, becoming a new interactive material.

\subsection{The correlation between the Picture \& Title Related and the release time of the song lists can significantly predict the listening effect.}

The song list picture is related to the title content, which can attract the user visually to a certain extent and increase the play of the song list. The picture is related to the title, conveying the overall information and emotional atmosphere of the song list, satisfying the user's needs for auditory senses and visual senses.

The publication of song list has significant predictive power for listening effect. With the extension of playback time, the amount of playback will continue to accumulate and increase. When choosing a song list, users will be influenced by word-of-mouth and evaluation. The amount of play and comment are the factors that evaluate the monument. The larger the volume of songs, the abler to attract more users.

\subsection{There is a significant positive correlation between the type of songs and the communication bias of the title.}

The assignment of songs is based on the mental state from tension to relaxation. Assignment is work $=1$, learning $=2$, travel $=3$, sports $=4$, night $=5$. There is a positive correlation between the title spread bias and the singing scene, which shows that users are more inclined to increase the reception of information and 
Citation: Lu Jiang and Wenying Wu, 2019. Research on the Factors Affecting the Communication Effect of Scene Music Song Lists. Journal of Applied Sciences Research., 15(5): 1-6. DOI: $10.22587 /$ jasr.2019.15.5.1

emotion in the relaxed mental state of the scene. Intense mental situations, such as work and study, users tend to reduce the amount of information and emotion they receive. In the tense situation between the two titles of information and emotion, users tend to spread the information bias of the song title.

\subsection{Song lists scene type has a certain impact on the number of songs and song language}

Users in a relaxed state of mind, such as night scenes, do not have much demand for songs. The list of songs is a presentation of musical information. The excessive number of songs will increase the burden on users to choose music information, and can not meet users' leisure needs.

The song language embodies the approximation between the music language and the user, and the adaptability of the music and the singing scene in different cultures.

\section{CONCLUSION}

This study analyzes the relationship between scene type and picture type, picture and title related, song number, song language, song list release time, song list description text, title propagation bias and other variables.

The study also conducted a multi-layer regression analysis on the influencing factors of the song list's communication effect, including the song list producer's experience.

The research results show that the scene type of the song list has a positive correlation with the listening effect and interaction effect. The listening and interaction effects of song lists in different scenes are different. The type of scene does not reflect a significant predictive power of the effect of the communication. It can be seen that under the influence of many factors, the influence of scene type on the communication effect is not significant.

There is a positive correlation between the song list type and the title communication bias. Under different scene types, the user's demand for emotions and information transmitted by the title of the song list is different.

The release time of the song list has significant predictive power for both the listening effect and the interactive effect. The mechanism of the release duration is similar to the snowball effect. Over time, the accumulation of UGC content such as word-of-mouth and comments on song lists will attract more users to listen and interact.

Title communication bias and image type have significant predictive power for interaction.

The picture is related to the title and has a significant predictive effect on the listening effect.

The song list scene type has a certain influence on the number of songs and song language.

The results of the study will be helpful in the production of song list content on the mobile music app platform. In particular, the analysis of the content and form of the song list, as well as the headline bias in different scenarios, helps the user to better use the elements of the song list to achieve the desired interaction and listening effect.

\section{References}

[1] Lan Peng (2015.) Scene: New Elements of Media in the Mobile Age. Journalism Review, (3): 20--27.

[2] Citation Missing

[3] Wang Jun feng (2016) Scene Music: A New Mode of Music Communication Based on User Scenes. Media, (21) :66-68

[4] Citation Missing

[5] Peng Qing (2017), a new revolution in music communication based on fan economy in the scene era. Southeast Communication, (6):4-6

[6] P Millward \& P Widdop \& M Halpin (2017) 'Different Class'? Homophily and Heterophily in the Social Class Networks of Britpop. Cultural Sociology. 11 (3) :318-336

[7] Citation Missing

[8] Citation Missing

[9] Spring, Kenneth,(2006)The regularization of risk in music scenes. ProQuest Dissertations and Theses

[10] Taylor Jodie (2012) Scenes and sexualities: Queerly reframing the music scenes perspective. Continuum, 26 (1) :143-156

[11] China Internet Information Center (2018) the 41st Statistical Report on Internet Development in China

[12] WA Collins (1985) No Sense of Place: The Impact of Electronic Media on Social Behavior. Contemporary Sociology, 56 (1) :65-66

[13] R Scoble (2006) Age of Context: Mobile, Sensors, Data and the Future of Privacy

[14] BJ Blacking, How Musical Is Man? (1974). Univ of Washington Pr.

[15] C Driver \& A Bennett (2015) Music Scenes, Space and the Body. Cultural Sociology, 9 (1):99-115

[16] [0M Tironi (2012) Enacting Music Scenes: Mobility, Locality and Cultural Production. Mobilities, 7 (2) :185-210 